Alma Mater Studiorum - Università di Bologna DEPARTMENT OF ECONOMICS

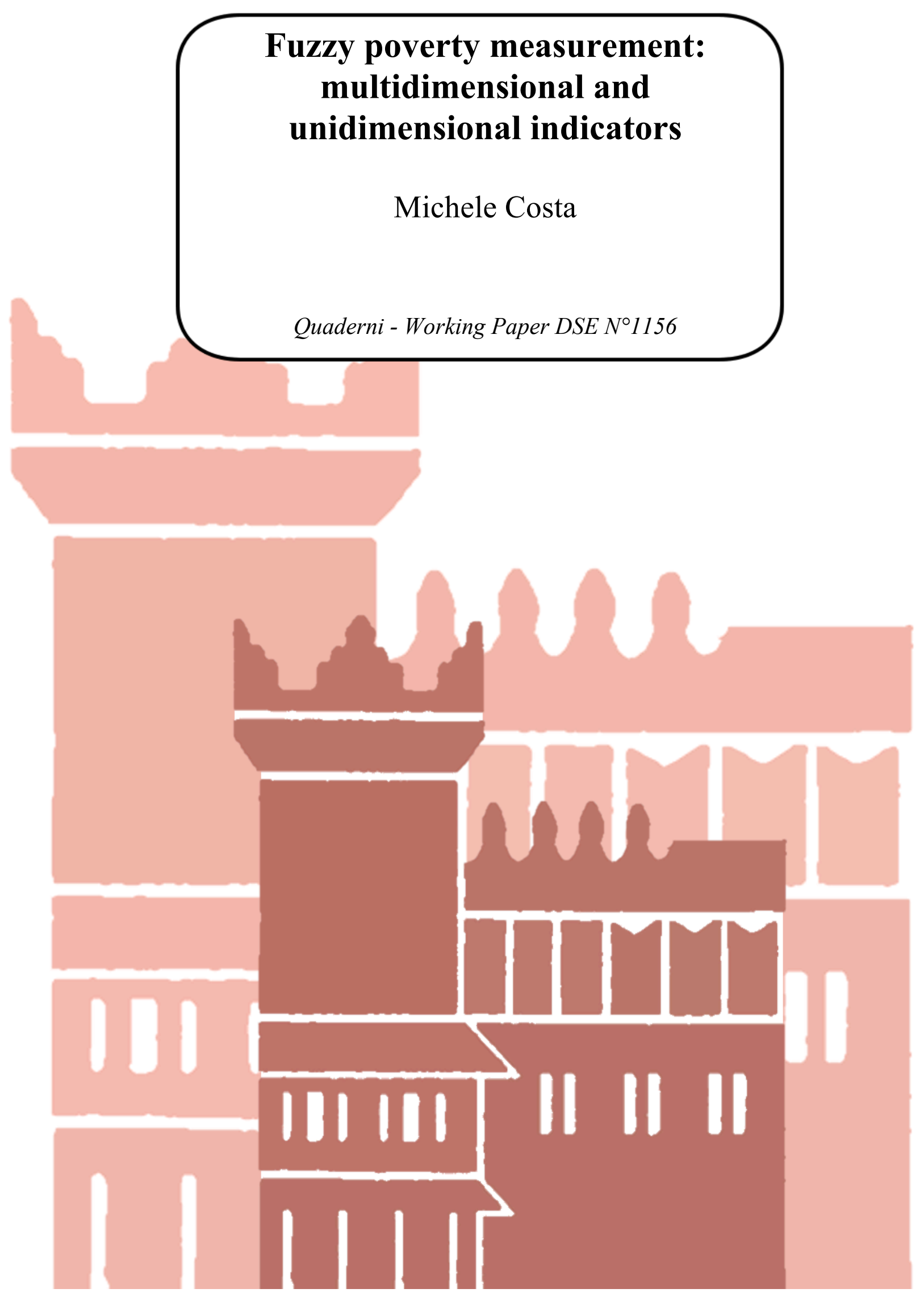




\title{
Fuzzy poverty measurement: multidimensional and unidimensional indicators
}

\author{
Michele Costa
}

\begin{abstract}
This paper proposes a comparison between multidimensional and unidimensional poverty indicators. Sets of poor units identified by traditional head count ratio, fuzzy unidimensional and fuzzy multidimensional indices are compared by means of a rank correlation analysis. The robustness of the comparison is ensured by a simulation study, which allows to address several issues related not only to fuzzy sets based methods, such as the subjective choice of membership to the poor set, but also to the multidimensional measurement, such as the effect of the weighting system. Our results stress that the unidimensional indicators provide partial information on poverty condition.
\end{abstract}

Key words: Multidimensional poverty index, Fuzzy indicators, Simulation study

JEL classification: D63; I32; C1

Michele Costa

Department of Economics, University of Bologna, e-mail: michele.costa@unibo.it 


\section{Non-technical summary}

Poverty is a multidimensional condition, not exclusively related to an insufficient provision of income or wealth, but the result of the contemporaneous occurrence of many factors. Multidimensional poverty is coherent with the concept of social exclusion, which is the target of modern welfare policies and which also represents a key element within the debate on alternative welfare measures to GDP.

Notwithstanding the general consensus about the multidimensional view of poverty, its quantitative measurement is frequently operated on the basis of a single indicator, usually the income, thus implying that the empirical assessments of unidimensional and multidimensional approach generally coincide.

In this case, the multidimensional approach would be an elegant and theoretically useful development, without the need to implement it empirically. Since the unidimensional indicator is extremely simpler and faster to calculate, if it is also fully informative, it represents a suitable solution for the measurement of poverty. When, on the other hand, the two approaches indicate two different sets of poor units, it is important to evaluate the different information contained in the two sets.

A relevant implication of the multidimensional approach is the opportunity to divide total population not simply into just two subgroups, poor and non poor, as it is done with poverty lines, but to detect a multiplicity of populations subgroups, each related to different poverty levels, gradually moving from the completely poor to the absolutely non poor condition.

A simple but effective method to detect different degrees of poverty, overcoming the dichotomy poor and non poor, is given by the fuzzy sets. Within fuzzy sets based methods it is possible to derive and to compose both multiple unidimensional and also multidimensional indicators, thus allowing a more general and flexible approach to poverty measurement.

In this work we aim to address some issues related to the choice of the degrees of membership to the poor of the different population subgroups: this choice belongs to the researchers and, therefore, is frequently view as arbitrary and questionable. We propose a simulation study in order to assess the effects on the set of poor units of different degrees of membership and to evaluate the robustness of the fuzzy poverty indicators.

We implement a rank correlation analysis able to provide an exhaustive comparison between the sets of poor units defined by the different methods. The result of rank correlation analysis allow to demonstrate that the two approaches define two different sets of poor households, and we also show how this difference increases as poorer population subgroups are targeted. Overall our results suggest not only two different theoretical frameworks, but also mismatched empirical findings, with the unidimensional indicator providing only partial information on poverty condition.

Relying on our conclusions, any socio-economic policy to reduce poverty developed on the basis of income information is likely to no achieve its proposed goals, being addressed to socioeconomic units which are, in effect, non-poor. Only in the framework of the multidimensional approach it is possible to correctly individuate the set of the poor and to formulate actions able to reduce poverty. 


\section{Introduction}

Poverty is a multidimensional condition, not exclusively related to an insufficient provision of income or wealth, but the result of the contemporaneous occurrence of many factors [12], [15]. Multidimensional poverty is coherent with the concept of social exclusion [1] [14], which is the target of modern welfare policies and which also represents a key element within the debate on alternative welfare measures to GDP.

Notwithstanding the general consensus about the multidimensional view of poverty, its quantitative measurement is frequently operated on the basis of a single indicator, usually the income, thus implying that the empirical assessments of unidimensional and multidimensional approach generally coincide.

In this case, the multidimensional approach would be an elegant and theoretically useful development, without the need to implement it empirically. Since the unidimensional indicator is extremely simpler and faster to calculate, if it is also fully informative, it represents a suitable solution for the measurement of poverty. When, on the other hand, the two approaches indicate two different sets of poor units, it is important to evaluate the different information contained in the two sets [5].

Our main contribution is to compare the sets of poor units defined by traditional unidimensional and multidimensional approach. For this purpose we develop a rank correlation analysis, able to investigate in depth the similarities between the different indicators.

A relevant implication of the multidimensional approach is the opportunity to divide total population not simply into just two subgroups, poor and non poor, as it is done with poverty lines, but to detect a multiplicity of populations subgroups, each related to different poverty levels, gradually moving from the completely poor to the absolutely non poor condition. Only 2 subgroups can represent a severe limit with just one inequality factor, but they are likely to be inadequate by using many inequality factors.

A simple but effective method to detect different degrees of poverty, overcoming the dichotomy poor and non poor, is given by the fuzzy sets. Within fuzzy sets based methods it is possible to derive and to compose both multiple unidimensional and also multidimensional indicators, thus allowing a more general and flexible approach to poverty measurement.

In this work we also aim to address some issues related to the choice of the degrees of membership to the poor of the different population subgroups: this choice belongs to the researchers and, therefore, is frequently view as arbitrary and questionable. The weakness implied by subjective choices refers to their influence on the stability of the results, that is, on the robustness of the method. Despite the variety of studies on multidimensional poverty, analyses on their robustness are still at a preliminary stage. Literature offers some interesting papers, among the others Alkire and Santos [2] analyze the effects on robustness related to a wide variety of elements, while Duclos et al. [10] develop a meaningful proposal in the framework of stochastic dominance. In the following we propose a simulation study in order to 
assess the effects on the set of poor units of different degrees of membership and to evaluate the robustness of the fuzzy poverty indicators.

Our purpose is to contribute to the theory of multidimensional poverty measurement by providing evidence in favor of fuzzy sets methods which we suggest as an efficient and rigorous solution. A second aim of the simulation study is to evaluate the effects of different sets of weights, first on the final indicator, and, most importantly, on the definition of the set of poor units. We implement the rank correlation analysis by referring also to the simulated indicators, thus providing an exhaustive comparison between the sets of poor units defined by the different methods. Besides the methodological aspects, our results are also interesting for applied poverty analysis and for poverty reduction policies, where the focus is the correct definition of the set of poor units.

The paper is organized as follows. Section 2 critically introduces the basic of fuzzy poverty indicators, Section 3 illustrates the Monte Carlo study developed to assess the properties of fuzzy indicators, Section 4 compares poor sets defined by traditional, fuzzy unidimensional and fuzzy multidimensional indicators, Section 5 presents a case study on Italian data and Section 6 concludes.

\section{Fuzzy poverty indicators}

Many Authors proposed, generalized or applied fuzzy sets based methods to the poverty analysis, among the others [7], [4], [8], [13]. In the variety of the meaningful contributions, we make a specific reference to the totally fuzzy approach by Cerioli and Zani [6] and to the relative fuzzy approach by [7].

Let be $A$ the set of the $n$ units $\left\{a_{1}, a_{2}, \ldots, a_{n}\right\}, B$ the subset of the poor units, and $\mu(X)$ an indicator function, which determines the degree of membership of the $i$-th unit to the set $B$ with respect to a certain attribute $X$.

The indicator function transforms the achievement of a unit with respect to $X$ to the deprivation of that unit. A low or null achievement implies a maximum deprivation, that is $\mu(X)=1$, while a high achievement leads to an absence of deprivation, that is $\mu(X)=0$. Traditionally $\mu(X)$ can assume only two values, 1 for the poor units and 0 for the non poor units, and $X$ is the (equivalent) income; the well known head count ratio $H$ is the most famous example of this case, obtained when the population is divided in two non overlapping subgroups, poor and non poor, on the basis of a poverty line $z$ which uniquely defines the subset of poor units $B$ :

$$
\mu(X)=\left\{\begin{array}{l}
\mu_{1} \text { with } \mu_{1}=1 \text { if } X<z \\
\mu_{2} \text { with } \mu_{2}=0 \text { if } X \geq z
\end{array}\right.
$$

and

$$
B=\bigcup\left(a_{i} \mid \mu(X)_{i}=1\right) .
$$

We obtain the head count ratio $H$ as the ratio between the number of poor and the size of the population 


$$
H=\sum_{i=1}^{n} \mu(X)_{i} n_{i} / \sum_{i=1}^{n} n_{i}
$$

where $\mu(X)_{i}$ and $n_{i}$ are the value of the indicator function and the sample weight of the $i$-th unit, respectively.

The presence of only two groups and the use of a poverty line $z$ introduce a high rigidity in the analysis, since, given an $\varepsilon$ as small as desired, any unit with income $x_{i}=z-\varepsilon$ is considered as a poor unit, while a unit with income $x_{i}=z+\varepsilon$ is classified as a non poor unit. In order to overcome this limit, which also strongly affects policies and actions aimed to poverty reduction, it is necessary a more flexible approach, such as the fuzzy method, able to allow more than two groups.

\subsection{Fuzzy unidimensional poverty indicators}

Within the fuzzy sets, $\mu(X)$ is no longer only equal to 0 or 1 , but ranges between 0 and 1 , thus allowing intermediate or partial membership to $B$ : if $\mu(X)=1$ and $\mu(X)=0$ still indicate a poor and a non poor unit, respectively, values as $\mu(X)=$ 0.8 and $\mu(X)=0.2$ refer to an almost poor and to an almost non poor unit, and $\mu(X)=0.5$ to a neither poor or non poor unit.

The first problem encountered in the framework of fuzzy indicators concerns the choice of the number of groups to consider: while, in the classic case, two groups, poor and non-poor, represent a forced choice, moving to $k>2$ groups $A_{1}, A_{2}, \ldots, A_{k}$ with $A=\cup A_{j}$ and $A_{j} \cap A_{h}=0 \quad \forall i, j$, the choice of $k$ depends on the preferences of the researchers.

In order to obtain the groups, the possible outcomes of $X$, ranging between $X_{\text {min }}$ and $X_{\text {max }}$, are divided in $k$ intervals, one for each of the $k$ groups; the intervals are defined by a set of $(k-1)$ values $\left\{X_{1}, X_{2}, \ldots, X_{k-1}\right\}$, chosen by the researchers with $X_{\text {min }}<X_{1}<X_{2}<\ldots<X_{k-1}<X_{\text {max }}$.

The poverty attribute $X$ can be a polytomous or a continuous variable (such as educational level or income) or also a composite indicator specifically derived by the researchers combining different variables (such as ownership of the dwelling, number of rooms and presence of toilet).

The second issue related to fuzzy indicators refers to the necessity to associate to each group a value of the indicator function, which therefore assumes $k$ values $\left\{\mu_{1}, \mu_{2}, \ldots, \mu_{k}\right\}$.

In this way the indicator function takes on a more complex form than in (1) and can be represented as

$$
\mu(X)=\left\{\begin{aligned}
& \mu_{1} \text { with } \mu_{1}=1 \text { if } X \in\left[X_{\text {min }}, X_{1}\right] \\
& \mu_{2} \text { if } X \in\left[X_{1}, X_{2}\right] \\
& \cdots \text { if } X \in\left[X_{k-2}, X_{k-1}\right] \\
& \mu_{k-1} \text { with } \mu_{k}=0 \text { if } X \in\left[X_{k-1}, X_{\text {max }}\right] . \\
& \mu_{k}
\end{aligned}\right.
$$


A fuzzy unidimensional index evaluates the poverty condition with respect to a certain attribute $X$ and is obtained as in (4):

$$
I(X)=\sum_{i=1}^{n} \mu(X)_{i} n_{i} / \sum_{i=1}^{n} n_{i} .
$$

where the main innovation is that $\mu(X)$ can also assume intermediate values between 0 and 1 . The head count ratio $H$ is a special case of $I(X)$, obtained when $k=2$ and the indicator function can be only 0 or 1 .

The fuzzy unidimensional index $I(X)$, as the head count ratio, ranges between 0 and 1 , and indicates the fraction of poor units on the total.

The fuzzy approach does not possess a poverty line partitioning total population into poor and non-poor; in order to identify poor units, it is sufficient to have the list of the $\mu(X)_{i}$ in decreasing order and to obtain its cumulative distribution $F(\mu(X))$ : if $F\left(\mu(X)_{i}\right)<I(X)$ we have a poor household, while $F\left(\mu(X)_{i}\right) \geq I(X)$ indicates a non-poor household.

The subset of poor units $B$ is given by the union of the subgroups $A_{j}$ associated to the highest values of $\mu(X)$ from $A_{1}$ up to the subgroup $A_{l}$ to which belongs the unit which fulfills the condition $F\left(\mu(X)_{i}\right)=I(X)$ :

$$
B=\bigcup_{j=1}^{l} A_{j} .
$$

Since this unit is usually not the last of its group, also in the unidimensional fuzzy framework we face a awkward situation: to the same subgroup $A_{l}$ belong poor units up to the $i$-th and non poor units from the $i$-th.

A finer classification with respect to only two cases, 0 and 1 , is a relevant improvement, but the choice of the intermediate values depends on the individual researchers, thus introducing into the analysis a certain degree of subjectivity and, potentially, of uncertainty and unstability.

Overall, in the analysis of fuzzy unidimensional indicators, we face two sources of subjectivity, the choice of $k$ and of the values $\left\{\mu_{2}, \ldots, \mu_{k-1}\right\}$ and it is therefore relevant to assess the impact of both these elements on the final indicator.

\subsection{Fuzzy multidimensional poverty indicators}

Moving from the unidimensional to the multidimensional framework requires to identify the different inequality factors and to define their number $m$, thus obtaining the structural economic, demographic and social factors $\left\{X_{1}, X_{2}, \ldots, X_{m}\right\}$ related to some form of social exclusion and able to describe and interpret the poverty condition.

Furthermore, within a multidimensional framework, it is essential to derive a weighting system which allows to rank the $m$ inequality factors. 
The presence of $m$ inequality factors leads to introduce a second subscript $j$, where $\mu\left(X_{j}\right)_{i}$ denotes the degree of membership to $B$ of the $i$-th unit with respect to the $j$-th inequality factor.

Furthermore, $m$ inequality factors imply $m$ indicator functions

$$
\mu\left(X_{j}\right)=\left(\mu_{j 1}, \mu_{j 2}, \ldots, \mu_{j k_{j}}\right) \quad j=1, \ldots, m
$$

where $k_{j}$ is the number of groups identified for the $j$-th inequality factor.

The result of the matching between the $n$ units and the $m$ indicator functions is a matrix $M(X)$ with $n$ rows and $m$ columns, where the generic element represents the degree of membership to the subset of poor units for the $i$-th unit with respect to the $j$-th factor.

By columns of the matrix $M(X)$, we derive the unidimensional poverty indices $I\left(X_{1}\right), I\left(X_{2}\right), \ldots, I\left(X_{m}\right)$ where

$$
I\left(X_{j}\right)=\sum_{i=1}^{n} \mu\left(X_{j}\right)_{i} n_{i} / \sum_{i=1}^{n} n_{i}
$$

measures the poverty level, that is the fraction of poor units, with respect to the $j$-th inequality factor.

By rows of $M(X)$, we obtain a multidimensional index for the $i$-th unit, $I\left(a_{i}\right)$, which measures the multidimensional degree of membership to the subset of poor units for the $i$-th unit.

$I\left(a_{i}\right)$ is obtained as a weighted sum of the $\mu\left(X_{j}\right)_{i}$

$$
I\left(a_{i}\right)=\sum_{j=1}^{m} \mu\left(X_{j}\right)_{i} w_{j} / \sum_{j=1}^{m} w_{j} .
$$

and ranges between 0 , when the $i$-th unit is considered non-poor with respect to all $m$ inequality factors, and 1 , when the $i$-th unit is poor with respect to all $m$ factors.

\subsubsection{The weighting structure}

The weights $w_{j}$ represent the distinctive feature of the multidimensional indicators and measure the intensity of deprivation and social exclusion related to the $j$-th inequality factor: the underlying guideline is that a factor not possessed by any unit has no effect on the social exclusion, while, on the contrary, if all the units but a few possess the factor, it represents a relevant source of inequality and social exclusion. A weighting system consistent with that is [6]

$$
w_{C Z j}=\log \left(n / \sum_{i=1}^{n} \mu\left(X_{j}\right)_{i} n_{i}\right)
$$

where $w_{j}$ is equal to 0 for $\mu\left(X_{j}\right)_{i}=1, i=1, \ldots, n$, while $w_{j}$ increases for decreasing $\sum_{i=1}^{n} \mu\left(X_{j}\right)_{i}$. 
The case $w_{j}=0$ deserves a special attention: it corresponds to the exclusion of the $\mathrm{j}$-th inequality factor $X_{j}$ and implies an indicator function for $X_{j}$ which is always equal to 1 for all units, that is: $\mu\left(X_{j}\right)_{1}=\mu\left(X_{j}\right)_{2}=\ldots=\mu\left(X_{j}\right)_{n}=1$. When $\mu\left(X_{j}\right)_{i}=$ $1 \forall i$, we assume that all units have the same value of $X_{j}$ and that all perceive the same degree of poverty and social exclusion with respect to $X_{j}$. Furthermore, since $\mu\left(X_{j}\right)_{i}=1$, the same degree of poverty shared by all units with respect to $X_{j}$ is the maximum: all are equally and most poor. If, for example, we do not consider the educational level, we implicitly assume that all the units have the same qualification, and that this qualification is also as low as possible: for the educational level it means to consider all units as without any educational achievement, which clearly represents a strongly unrealistic assumption.

The effects of $w_{j}=0$ are frequently overlooked, while they have a relevant impact, which we need to take into account.

A meaningful review of the issues related to the weighting structure is proposed by [11], who stress both the relevance and the subtleness of this topic.

In order to assess the role and the effects of the weighting structure, the proposal outlined in (6) can be compared to other contributions, first of all an equally weighted index where $w_{e j}=1 / m \forall j$.

A further set of weights can be derived from the budget allocation technique, where the importance of the different factors is given by their budget share.

A final proposal worth of attention is the structure $w_{U N}$ adopted by the Human Development Index [16], which assigns the same weight, 0.33, to the educational domain, the wealth domain, and the living conditions domain, and, within domains, equal weight across indicators.

In the following we develop the comparison of the unidimensional indicator with the multidimensional index on the basis of different weighting systems, namely $w_{e}$, $w_{U N}$ and $w_{U N}$.

\subsubsection{The multidimensional poverty index for the population}

The synthesis of the $m$ unidimensional indices $I\left(X_{j}\right)$ and of the $n$ multidimensional indices $I\left(a_{i}\right)$ in a multidimensional poverty index for the population can be achieved quite simply by means of a weighted sum

$$
I=\frac{\sum_{j=1}^{m} I\left(X_{j}\right) w_{j}}{\sum_{j=1}^{m} w_{j}}=\frac{\sum_{i=1}^{n} I\left(a_{i}\right) n_{i}}{\sum_{i=1}^{n} n_{i}}=\frac{\sum_{j=1}^{m} w_{j} \sum_{i=1}^{n} \mu\left(X_{j}\right)_{i} n_{i}}{\sum_{j=1}^{m} w_{j} \sum_{i=1}^{n} n_{i}}
$$

As in the fuzzy unidimensional case, also the multidimensional approach does not possess a poverty line and poor households are still identified on the basis of the cumulative distribution of the $I\left(a_{i}\right)$ in descending order: if $F\left(I\left(a_{i}\right)\right)<I$ we have a poor household, while $F\left(I\left(a_{i}\right)\right) \geq I$ indicates a non-poor household.

The definition of the set of the poor units $B$ is a little more complicated in the multidimensional framework. First, for each poverty attribute $X_{j}$ is obtained $B_{j}$, the set of poor units with respect to $X_{j}$. Second, the set $B$ is given by the intersection 
of $B_{1}, B_{2}, \ldots, B_{m}$, or, more frequently, by the union of the intersections of different combinations of the $B_{j}$ :

$$
B=\left(\cap B_{j}\right) \cup\left(\cap B_{h}\right) .
$$

Since the multidimensional indicator $I$ is also a function of the weight $w_{j}$ it is not possible to know ex ante the exact list of $B_{j}$ which gives $B$. This is not to be perceived as a limit, but, on the contrary, as an opportunity of fuzzy multidimensional indicators, which take advantage of the flexibility ensured by the $\alpha$ cut method, where we refer to a certain amount $\alpha$ of the population: $F\left(I\left(a_{i}\right)=\alpha\right.$. By targeting a given fraction of the poorest unit, we obtain $B_{\alpha}=F\left(I\left(a_{i}\right)=\alpha\right.$ and the relevant inequality factors which determine $B_{\alpha}: B_{\alpha}=U B_{j}$. The combination of factors at the basis of $B_{\alpha}$ is not necessarily the same over $\alpha$, but it provides powerful insights on the inequality structure and a strong support to any social and economic policy for poverty reduction.

The issue of the subjectivity related to the choice of $\mu_{1}, \mu_{2}, \ldots, \mu_{k}$, anticipated in the previous section for the unidimensional indicator, is amplified and more urgent in the multidimensional framework, where we have $m$ indicator functions to determine. In order to investigate this point, which is frequently perceived as one of the weaknesses of fuzzy sets based indicators, in the following we develop a simulation study.

\section{Robustness of fuzzy indicators: a simulation study}

With the aim to assess the degree of robustness of the method and to evaluate the effects of $\mu(X)$ on the poverty index, we propose a simulation study where the scores of the indicator function are not a priori chosen, but endogenously determined by means of a Monte Carlo experiment.

We randomly extract $\mu(X)$ from an uniform distribution within a set of $k$ intervals determined according to the following scheme:

$$
\mu\left(X_{j}\right)=\left\{\begin{array}{c}
\mu_{j 1} \text { with } \mu_{j 1} \in\left[\mu_{j 2}+h, 1\right] \\
\ldots \\
\mu_{j k-1} \text { with } \mu_{j k-1} \in\left[\mu_{j k_{j}}+h, 2 / k_{j}\right] \\
\mu_{j k} \text { with } \mu_{j k_{j}} \in\left[0,1 / k_{j}\right]
\end{array}\right.
$$

where $k_{j}$ is the exogenous number of classes proposed for $X_{j}$ and $h$ is an exogenous minimum distance between two different values of $\mu(X)$.

By setting, e.g., $k=45$ and $h=0.1$, on the basis of (8) we obtain

$$
\mu\left(X_{j}\right)=\left\{\begin{array}{l}
\mu_{j 1} \text { with } \mu_{j 1} \in\left[\mu_{j 2}+0.1,1\right] \\
\mu_{j 2} \text { with } \mu_{j 2} \in\left[\mu_{j 3}+0.1,0.80\right] \\
\mu_{j 3} \text { with } \mu_{j 3} \in\left[\mu_{j 4}+0.1,0.60\right] \\
\mu_{j 4} \text { with } \mu_{j 4} \in\left[\mu_{j 5}+0.1,0.40\right] \\
\mu_{j 5} \text { with } \mu_{j 5} \in[0,0.20]
\end{array}\right.
$$


that is we extract $\mu_{15}$, the degree of membership to the richest subgroup, from an uniform distribution within the interval $[0,0.20]$, then we move to $\mu_{14}$, which belongs to the interval $\left[\mu_{14}+0.1,0.4\right]$, and so on, up to $\mu_{j 1}$, which is randomly extracted from an uniform distribution within the interval $\left[\mu_{12}+0.1,1\right]$.

This scheme represents a perhaps excessive generalization, since the first and last values, namely the two associated to the poorest and to the richest group, respectively, are almost always 1 and 0 , and therefore should not be included in the simulation. By setting $\mu_{j 1}=1$ and $\mu_{j k}=0$, the simulation study refers to the remaining $k-2$ values of the $\mu(X)$ function, following a scheme such as

$$
\mu\left(X_{j}\right)=\left\{\begin{aligned}
\mu_{j 1} \text { with } \mu_{j 1}=1 \\
\mu_{j 2} \text { with } \mu_{j 2} \in\left[\mu_{j 3}+h,(1-h)\right] \\
\ldots \\
\mu_{j k-2} \text { with } \mu_{j k-2} \in\left[\mu_{j k-1}+h, 2(1-h) /(k-2)\right] \\
\mu_{j k-1} \text { with } \mu_{j k-1} \in[h,(1-h) /(k-2)] \\
\mu_{j k} \text { with } \mu_{j k}=0
\end{aligned}\right.
$$

By referring to the new scheme (10), where $\mu_{j 1}=1$ and $\mu_{j k}=0$, the previous example, with $k=5$ and $h=0.1$, results:

$$
\mu\left(X_{j}\right)=\left\{\begin{array}{l}
\mu_{j 1} \text { with } \mu_{j 1}=1 \\
\mu_{j 2} \text { with } \mu_{j 2} \in\left[\mu_{j 3}+0.1,0.9\right] \\
\mu_{j 3} \text { with } \mu_{j 3} \in\left[\mu_{j 4}+0.1,0.60\right] \\
\mu_{j 4} \text { with } \mu_{j 4} \in\left[\mu_{j 5}+0.1,0.30\right] \\
\mu_{j 5} \text { with } \mu_{j 5}=0
\end{array}\right.
$$

The main purpose of our Monte Carlo experiment, achieved by comparing exogenous schemes as in (3) to simulated schemes as in (10), is to investigate the stability of the fuzzy poverty indices and to evaluate their robustness. By means of the same experiment we are also able to investigate other aspects of fuzzy methods. First of all we analyze the effects related to the number of groups $k_{j}$ for the each of the $m$ inequality factors, second we study the role of $h$, that is the distance between consecutive values of the indicator function.

\section{Unidimensional and multidimensional comparison}

The key point of the comparison between the unidimensional and the multidimensional approaches refers to the set B of poor units and, in particular, to the evaluation of the intersection between the two sets.

The reference to the set $\mathrm{B}$ of poor units is useful also in order to evaluate the effects of different specifications of the $\mu(X)$ : to the extent that different $\mu(X)$ modify, or not, the set $\mathrm{B}$, we derive an indication on the robustness of the multidimensional indicators. A similar pattern can be followed in order to assess the effects of al- 
ternative weighting systems and of various compositions of the multidimensional index.

From the unidimensional approach, as well as from the multidimensional one and from the simulations, we first obtain a list of the units, from the poorest to the richest.

Second, we carry out a correlation analysis on the ranks $u_{i}$ of the list obtained from the unidimensional approach and on the ranks $v_{i}$ of the list resulted from the multidimensional approach. Later, the same procedure is used for comparing the lists obtained during the simulation with different $\mu(X)$.

Rank correlation analysis allows to effectively compare the two lists, and also to investigate the similarity of specific subgroups of units, which is particularly useful in our case, as we aim to the comparison between the subgroups of the poor units $B$ and also between subsets of $B$, that is $B_{\alpha}$.

The first standard correlation measure used is the Bravais-Pearson correlation coefficient $r$ applied to the ranks $\left(u_{i}, v_{i}\right)$ :

$$
r=\frac{\sum_{i=1}^{n}\left(u_{i}-\bar{u}\right)\left(v_{i}-\bar{v}\right)}{\sum_{i=1}^{n}\left(u_{i}-\bar{u}\right)^{2} \sum_{i=1}^{n}\left(v_{i}-\bar{v}\right)^{2}}
$$

where $\bar{u}$ and $\bar{v}$ are, respectively, the mean of $u_{i}$ and the mean of $v_{i}$.

The second correlation measure is represented by Kendall's $\tau$, which can be expressed as

$$
\tau=\frac{C-D}{n(n-1) / 2}
$$

where $C$ is the number of concordant pairs of ranks $\left(u_{i}, v_{i}\right)$ and $D$ is the number of discordant pairs of ranks $\left(u_{i}, v_{i}\right)$. Kendall's index does not consider the amount of differences between $u_{i}$ and $v_{i}$ and therefore can be less informative than other measures, such as Bravais-Pearson $r$.

A further important rank correlation measure is represented by the Spearman index $S$ :

$$
S=\frac{1-6 * \sum_{i=1}^{n}\left(u_{i}-v_{i}\right)^{2}}{n\left(n^{2}-1\right)}
$$

Finally, the fourth correlation measure considered is the Gini rank correlation, or cograduation, index $G$

$$
G=\frac{\sum_{i=1}^{n}\left|u_{i}-v_{i}^{\prime}\right|\left|u_{i}-v_{i}\right|}{n^{2} / 2}
$$

where $v_{i}^{\prime}$ is the rank of the multidimensional list by ascending order, that is from the richest to the poorest.

All indices range between -1 and 1 , reaching their maximum (minimum) value for perfect positive (negative) correlation and assuming value 0 for the absence of correlation between $u_{i}$ and $v_{i}$.

A relevant improvement of the information provided by the correlation indices refers to the number of the units involved in the analysis. First, we start including all units into the calculus of the indices, thus achieving a general evaluation related 
to the overall population. In the following step we restrict the correlation analysis to a fraction $\alpha$ of the available units, thus focusing on specific subsamples of the population, in primis the set $B$ of the poor units. Since our main interest is related to the comparison between the sets of poor units suggested by different methods, this focus is extremely appealing and allows a powerful insight on the robustness of fuzzy indicators.

Furthermore, in order to compare the two sets of poor units defined by uni- and multidimensional approaches, it is also possible to derive a similarity index. Our information set is given by the equivalent income $X_{1}$, the poverty line $z$, the set of poverty units defined by $X_{1}, B_{u}$, its size $n_{B u}$, the multidimensional poverty index $I$, the set of poverty units defined by $I, B_{m}$, and its size, $n_{B m}$.

Starting from the unidimensional approach, a similarity index $S_{u}$ can be obtained as the ratio between the number of poor in the multidimensional approach with income lower than the poverty line and the number of poor in the unidimensional approach:

$$
\begin{gathered}
s_{i}=\left\{\begin{array}{l}
0 \text { if } x_{i} \geq z \\
1 \text { if } x_{i}<z \text { and } i \in B_{m}
\end{array}\right. \\
S_{u}=\sum_{i=1}^{n_{B m}} s_{i} n_{i} / n_{B u}
\end{gathered}
$$

It is also possible to generalize $S_{u}$ by referring not to the standard poverty line $z$, but to a set of poverty lines $z_{\alpha}$ which define the $\alpha \%$ poorest of the observations in the unidimensional approach.

On the basis of the multidimensional approach, a similarity index $S_{m}$ first requires to introduce a poverty line $z^{*}$, which can be set equal to the income $x^{*}$ of the richest unit among the poor units in the multidimensional approach. Then we can obtain $S_{m}$ as the ratio between the number of poor in the unidimensional approach with income lower than $z^{*}$ and the number of poor in the multidimensional approach:

$$
\begin{gathered}
s_{i}=\left\{\begin{array}{l}
0 \text { if } x_{i} \geq z^{*} \\
1 \text { if } x_{i}<z^{*} \text { and } i \in B_{u}
\end{array}\right. \\
S_{m}=\sum_{i=1}^{n_{B u}} s_{i} n_{i} / n_{B m}
\end{gathered}
$$

Analogously to $S_{u}$, we can generalize also $S_{m}$ by referring to poverty lines $z_{\alpha}^{*}$, which define the $\alpha \%$ of the poorest of the observations in the multidimensional approach.

\section{Data and results}

In order to analyze the robustness of fuzzy sets based methods and to compare unidimensional and multidimensional poverty indicators we resort to the Italian house- 
holds data for the 2016. We illustrate the advantages of fuzzy indicators starting from the unidimensional case by comparing the traditional head count ratio to a fuzzy indicator and to a simulated case. By adding further inequality factors we develop a fuzzy multidimensional poverty indicator and we analyze its robustness. Finally we provide a comparison between uni- and multidimensional poverty indicators by means of a rank correlation analysis.

\subsection{Data}

The Survey on Households Income and Wealth performed by the Bank of Italy provides a powerful database which allows to derive a wide set of both simple and composite indicators based on individual and household information.

To the first set of indicators, related to the wealth domain, belong the household equivalent income (I), the ratio between the number of the household members perceiving an income and the household size (II), the value and the occupancy title of the household residence (III).

We also include two indicators concerning the educational domain: the educational achievement of the household head and his/her father (IV), and the educational achievement of the household spouse and her/his father (V).

The further 5 indicators assess the living conditions domain. First a composite indicator given by gender, age and occupational status of the household head (VI), second the geographical area of residence (VII), third the household size and number of bathrooms of the residence (VIII), fourth the household size and dimension of the residence (IX), fifth the job status of the household head and the other components of the household $(\mathrm{X})$.

\subsection{Simulation results}

Our first step in the presentation of the results refers to the effects on the fuzzy indicators related to $k$, the number of subgroups and $h$, the distance between two consecutive values of $\mu(X)$.

In Table 1 are shown some summary descriptive statistics related to 10000 simulations of the unidimensional fuzzy indicator $I(X)$, as in (4), computed for each combination of $k$ and $h$. The values of $\mu(X)$ are obtained as in (10), and the $n$ observations are randomly attributed to the $k$ classes. We present the results for $k=3$, $k=4$ and $k=5$ for the number of subgroups, for $h=0.05, h=0.10$ and $h=0.20$ for the distance between two consecutive values of $\mu(X)$ and we refer to $n=7421$ for the number of observations, that is the number of households in the 2016 Bank of Italy survey used in the following. 
Table 1 Mean $\mu$, standard deviation $\sigma$, skewness $\gamma_{1}$ and kurtosis $\gamma_{2}$ indices for 10000 simulated unidimensional fuzzy indicators $I(X)$ by $k$, number of subgroups, and $h$, minimum distance between two consecutive values of $\mu(X)$.

\begin{tabular}{llllllllllllll}
\hline$h$ & \multicolumn{3}{c}{$k=3$} & \multicolumn{4}{c}{$k=4$} & \multicolumn{4}{c}{$k=5$} \\
& $\mu$ & $\sigma$ & $\gamma_{1}$ & $\gamma_{2}$ & $\mu$ & $\sigma$ & $\gamma_{1}$ & $\gamma_{2}$ & $\mu$ & $\sigma$ & $\gamma_{1}$ & $\gamma_{2}$ \\
0.05 & 0.50 & 0.08 & 0.01 & -1.16 & 0.48 & 0,07 & -0.38 & -0.50 & 0.47 & 0.05 & -0.59 & 0.02 \\
0.10 & 0.50 & 0.08 & 0.00 & -1.19 & 0.48 & 0,05 & -0.39 & -0.46 & 0.48 & 0.04 & -0.61 & 0.05 \\
0.20 & 0.50 & 0.06 & 0.01 & -1.14 & 0.49 & 0,03 & -0.37 & -0.42 & 0.49 & 0.01 & -0.45 & 0.01 \\
\hline
\end{tabular}

As $h$, the minimum distance between two consecutive values of $\mu(X)$, increases, a decreasing standard deviation is observed, as expected, that is a lower variability of the simulated unidimensional fuzzy indices $I(X)$.

The variability of the simulated $I(X)$ is, as intuitively, also negatively affected by the number of subgroups $k$, which also influence the skewness $\gamma_{1}$ of the $I(X)$ : for $k=3$ we get a symmetric distribution, while for $k>3$ the scheme (10) leads to skewed distributions, with a fat tail for smaller values, i.e. for richer units. For $k=3$ there is an almost uniform distribution, while, as $k$ increases, there is both a decrease in the importance of the tails, and the presence of negative skewness.

In the following, we exploit the potential of scheme (10) with the aim of carrying out an in-depth analysis of the fuzzy indicators.

\subsection{Unidimensional poverty indicator}

The second step of our results concerns to the unidimensional poverty indicators, where we compare the traditional head count ratio to both a fuzzy income-based indicator and simulated fuzzy indicators.

We use the OECD equivalence scale and a poverty line $z$ being the $60 \%$ of the median $\bar{x}_{m e}$ of the equivalent income: $z=0.6 * \bar{x}_{m e}$.

An income based fuzzy poverty indicator, instead to divide total population in only two subgroups, is based on $k>2$ subgroups: for example

$$
\mu\left(X_{1}\right)=\left\{\begin{array}{l}
1.0 \text { if } x_{1 i}<0.4 \bar{x}_{1 m e} \\
0.9 \text { if } 0.4 \bar{x}_{1 m e} \leq x_{1 i}<0.6 \bar{x}_{1 m e} \\
0.5 \text { if } 0.6 \bar{x}_{1 m e} \leq x_{1 i}<0.8 \bar{x}_{1 m e} \\
0 \text { if } 0.8 \bar{x}_{1 m e} \leq x_{1 i}
\end{array}\right.
$$

In (15) we allow a greater flexibility with respect to (1): under the poverty line $z$ we assume two classes, introducing a difference among the poor, and also above the poverty line we differentiate among the non poor, assigning a positive membership $\left(\mu_{13}=0.5\right)$ to units with income below $0.8 \bar{x}_{1 m e}$.

In order to vary the scores of the indicator function $\mu\left(X_{1}\right)$, we run a Monte Carlo experiment, by using the scheme (10) with $k=4$ and $h=0.1$ : 


$$
\mu\left(X_{1}\right)=\left\{\begin{array}{l}
\mu_{11} \text { with } \mu_{11}=1 \\
\mu_{12} \text { with } \mu_{12} \in\left[\mu_{13}+0.1,0.9\right] \\
\mu_{13} \text { with } \mu_{13} \in[0.1,0.45] \\
\mu_{14} \text { with } \mu_{14}=0
\end{array}\right.
$$

Given the $\mu\left(X_{1}\right)$, we derive the fuzzy unidimensional indicator $I\left(X_{1}\right)$ as in (4), and we compare the results related to (15) and to (16), thus investigating the effects of $\mu(X)$ on the poverty indicator.

Table 1 reports in the first column the head count ratio, equal to 0.202 , in the second the fuzzy indicator based on (15), equal to 0.264 , while in the following columns are summarized the results of 100000 random extractions of $\mu\left(X_{1}\right)$ as in (16). We report, in the first row some values of $I\left(X_{1}\right)$, ranging from 0.14 to 0.25 , and from the third to the fifth row the mean of the respective simulated $\mu(X)$. It is possible to observe how extreme values of $I\left(X_{1}\right)$ occur only in correspondence with unlikely values of $\mu(X): I\left(X_{1}\right)=0.14$, for example, requires $\mu_{12}=0.30$, which is clearly an inadmissible value for incomes below the poverty line. When, however, $\mu(X)$ remains within acceptable ranges, the index $I\left(X_{1}\right)$ does not show significant changes.

Table 2 Head count ratio with $\mu(X)=(2)$, fuzzy poverty indicator with $\mu(X)=(5)$ and fuzzy poverty indicator with simulated $\mu(X)=(6)$, Italian households 2016

\begin{tabular}{llllllllll}
\hline$I\left(X_{1}\right)$ & 0.202 & 0.264 & 0.142 & 0.161 & 0.181 & 0.201 & 0.219 & 0.238 & 0.252 \\
$\mu\left(X_{1}\right)$ & $(1)$ & $(15)$ & $(16)$ & $(16)$ & $(16)$ & $(16)$ & $(16)$ & $(16)$ & $(16)$ \\
$\mu_{11}$ & 1.00 & 1.00 & 1.00 & 1.00 & 1.00 & 1.00 & 1.00 & 1.00 & 1.00 \\
$\mu_{12}$ & 1.00 & 0.90 & 0.300 & 0.420 & 0.535 & 0.650 & 0.725 & 0.813 & 0.879 \\
$\mu_{13}$ & 0 & 0.50 & 0.133 & 0.170 & 0.212 & 0.257 & 0.331 & 0.389 & 0.434 \\
$\mu_{14}$ & 0 & 0 & 0 & 0 & 0 & 0 & 0 & 0 & 0 \\
\hline
\end{tabular}

We repeat the same procedure also for the other indicators, still obtaining similar results: the scores of the indicator functions $\mu\left(X_{j}\right)$ and the values of the unidimensional fuzzy indicators $I\left(X_{j}\right)$ are validated through a Monte Carlo study that ensures their robustness.

The adopted values of the indicator functions $\mu\left(X_{j}\right)$ are shown in the Appendix for the entire set of indicators used in the paper, without adding the simulations results, which would only repeat the scheme presented in this paragraph for the first indicator.

Overall, we derive a set of 10 unidimensional fuzzy indicators which provide a powerful basis for the development of the multidimensional index. 


\subsection{Multidimensional poverty indicator}

The multidimensional fuzzy poverty index $I$, obtained as (7), is a function of unidimensional fuzzy indicators and weights.

Our first goal is to evaluate the effects of the weighting system on the multidimensional indicator, for this purpose we analyze three different types of weights: the case of equal weights $w_{e}$, the structure adopted by the United Nations $w_{U N}$, a system based on social exclusion and proposed by Cerioli and Zani $w_{C Z}$.

In Table 3 are reported, together with the 10 unidimensional indices $I(X)$, the results for the three (normalized) weighting systems and the related multidimensional fuzzy poverty index $I$ as in (7).

Table 3 Unidimensional poverty indicators, weights and multidimensional poverty index - Italian households 2016 .

\begin{tabular}{lllllllllllll}
\hline & \multicolumn{1}{c}{ unidimensional indices } & & \multicolumn{2}{c}{ multidim. } \\
& I & II & III & IV & V & VI & VII & VIII & IX & X & index \\
\hline$I(X)$ & 0.264 & 0.037 & 0.163 & 0.341 & 0.184 & 0.033 & 0.128 & 0.094 & 0.329 & 0.306 & \\
$w_{e}$ & 0.10 & 0.10 & 0.10 & 0.10 & 0.10 & 0.10 & 0.10 & 0.10 & 0.10 & 0.10 & 0.188 \\
$w_{U N}$ & 0.111 & 0.111 & 0.111 & 0.167 & 0.167 & 0.067 & 0.067 & 0.067 & 0.067 & 0.067 & 0.199 \\
$w_{C Z}$ & 0.069 & 0.170 & 0.094 & 0.056 & 0.088 & 0.176 & 0.106 & 0.122 & 0.058 & 0.061 & 0.144 \\
\hline
\end{tabular}

The unidimensional poverty indicators $I(X)$ in the first row of the Table indicate the fraction of poor units on the total according to the different inequality factors, ranging from $34.1 \%$ of the fourth indicator (educational achievement of the household head and his/her father) to $3.3 \% 3$ per cent of the sixth (gender, age, occupational status of the household head). Consistent with the intuition underlying the weights related to social exclusion, $w_{C Z}$, the higher weights correspond to the smaller indices.

The last column of the table shows the multidimensional index $I$, which is equal to $I_{e}=0.188$ by using equal weights, to $I_{U N}=0.199$ by referring to the United Nations weighting system, and to $I_{C Z}=0.144$ on the basis of the weights (6) related to social exclusion.

Results provided by Table 3 allow interesting insights on the poverty structure. Multiplying the unidimensional indices $I\left(X_{j}\right)$ in the first row by the weights and dividing by the multidimensional index $I$ in the last column, we obtain the quantity $I\left(X_{j}\right) w_{j} / I$, that is the influence of the single inequality factors on $I$.

The ratios $I\left(X_{j}\right) w_{j} / I$, together with the related indicator, are shown in descending order in Table 4 . The fourth indicator, educational achievement of the household head and his/her father, represents the main source of inequality, followed by a group of four indicators: dimension of the residence (IX), job status of the household head and the other components of the household (X), education of the spouse (V), equivalent income (I). The last 5 indicators in order of relevance are the value and the occupancy title of the household residence (III), the geographical area of residence 
(VII), the household size and number of bathrooms of the residence (VIII), the ratio between the number of the household members perceiving an income and the household size (II), gender, age and occupational status of the household head (VI).

Equal weights $w_{e}$ and social-exclusion related weights $w_{C Z}$ suggest the same ranking of the inequality factors, while on the basis of United Nations weights $w_{U N}$ we observe some differences from the second to the fifth position.

Overall, in the second panel of Table 4 we observe how the living conditions domain accounts for over 45 per cent of the inequality for $w_{e}$ and $w_{C Z}$, while for $w_{U N}$ the main source of inequality is the educational domain.

Table 4 Ratios $I\left(X_{j}\right) w_{j} / I$ and poverty indicator - Italian households 2016.

\begin{tabular}{llllll}
\hline$I\left(X_{j}\right) w_{e j} / I_{e}$ & \multicolumn{2}{l}{$I\left(X_{j}\right) w_{U N j} / I_{U N}$} & \multicolumn{2}{l}{$I\left(X_{j}\right) w_{C Z j} / I_{C Z}$} \\
\hline 0.181 & IV & 0.286 & IV & 0.133 & IV \\
0.175 & IX & 0.155 & V & 0.133 & IX \\
0.163 & X & 0.148 & I & 0.130 & X \\
0.141 & I & 0.111 & IX & 0.127 & I \\
0.098 & V & 0.103 & X & 0.113 & V \\
0.087 & III & 0.091 & III & 0.107 & III \\
0.068 & VII & 0.043 & VII & 0.094 & VII \\
0.050 & VIII & 0.032 & VIII & 0.080 & VIII \\
0.020 & II & 0.021 & II & 0.044 & II \\
0.018 & VI & 0.011 & VI & 0.040 & VI \\
\hline 0.474 & living & 0.441 & education & 0.477 & living \\
0.279 & education & 0.299 & living & 0.277 & wealth \\
0.247 & wealth & 0.260 & wealth & 0.246 & education \\
\hline
\end{tabular}

In order to address the robustness issue for the multidimensional index, we further develop the simulation study, randomly extracting the indicator function $\mu\left(X_{j}\right)$ as in (10) for the ten fuzzy indicators and obtaining the multidimensional index on the basis of the three weighting system used in the paper.

The results related to 30000 simulated indices are illustrated in Figure 1 and indicate how weights have relevant effects on the multidimensional index: the use of $w_{C Z}$ leads to the smallest values of the index $I$, while the highest values are linked to $w_{U N}$, thus confirming the pattern already observed in Table 3 .

Besides the unidimensional fuzzy indices $I\left(X_{j}\right)$ and the multidimensional $I$, we also derive the list obtained from the unidimensional approach and the list resulted from the multidimensional approach, thus allowing a comparison between the two approaches. 


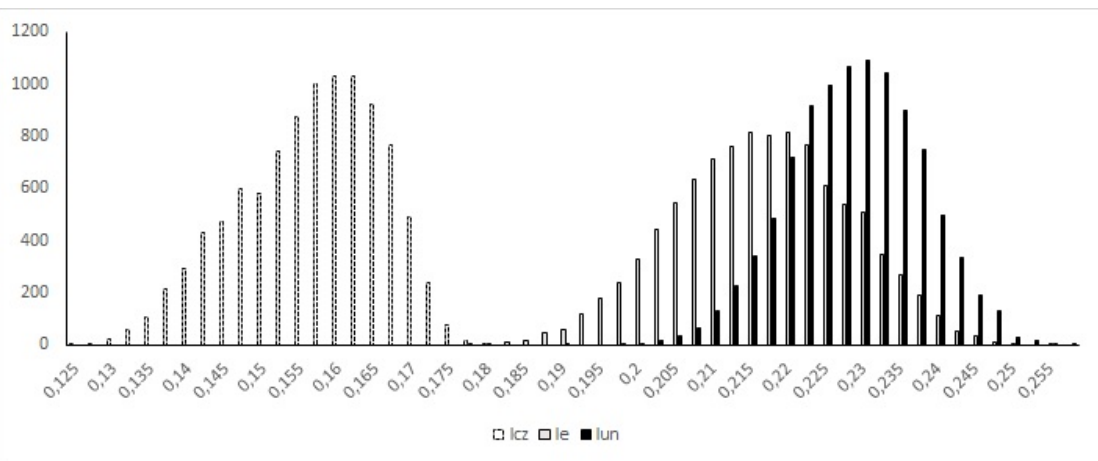

Fig. 1 Frequency histogram of 30000 simulated multidimensional fuzzy poverty indices by weights $w_{C Z}, w_{e}$ and $w_{U N}$.

\subsection{Unidimensional and multidimensional comparison}

In order to compare the unidimensional and the multidimensional approaches, we start with Tables 5, which reports a subset of observations for the four variables of interest: the household equivalent income $X_{1 i}$, its rank $u_{i}$, the multidimensional poverty index $I\left(a_{i}\right)$, its rank $v_{i}$.

Table 5 Household equivalent income $X_{1 i}$, its rank $u_{i}$, multidimensional poverty index $I\left(a_{i}\right)$, its rank $v_{i}$ - Italian households 2016.

\begin{tabular}{llllllll}
\hline$u_{i}$ & $X_{1 i}$ & $v_{i}$ & $I_{i}$ & $v_{i}$ & $I_{i}$ & $u_{i}$ & $X_{1 i}$ \\
\hline 1 & -6763 & 3785 & 0.109 & 1 & 0.779 & 6 & 0 \\
10 & 0 & 7 & 0.706 & 10 & 0.667 & 336 & 4960 \\
25 & 0 & 128 & 0.463 & 25 & 0.605 & 87 & 1429 \\
50 & 0 & 1556 & 0.215 & 50 & 0.550 & 424 & 5840 \\
75 & 802 & 2890 & 0.145 & 75 & 0.515 & 21 & 0 \\
100 & 1667 & 301 & 0.383 & 100 & 0.493 & 960 & 8820 \\
7321 & 57052 & 5156 & 0.061 & 7321 & 0.00 & 6940 & 37333 \\
7346 & 62854 & 7160 & 0.012 & 7346 & 0.00 & 7182 & 45859 \\
7371 & 69769 & 7402 & 0.00 & 7371 & 0.00 & 7279 & 52070 \\
7396 & 85375 & 7407 & 0.00 & 7396 & 0.00 & 7343 & 62000 \\
7411 & 131831 & 7414 & 0.00 & 7411 & 0.00 & 7406 & 113684 \\
7421 & 218805 & 7421 & 0.00 & 7421 & 0.00 & 7421 & 218805 \\
\hline
\end{tabular}

First, we rank the observations on the basis of $u_{i}$, with the left part of Table 5 showing $X_{1 i}, u_{i}, I\left(a_{i}\right)$, and $v_{i}$ for a subset of selected observations. Observations presenting the same value of $X_{1 i}$ are sorted by decreasing size of the multidimensional indicator $I\left(a_{i}\right)$. By comparing column 2 to column 4 in Table 5 , we can observe a strong similarity between the highest ranks, while, on the contrary, the lowest ranks are quite different. 
Our second step is to rank the observations on the basis of $v_{i}$, with the right part of Table 5 showing $X_{1 i}, u_{i}, I\left(a_{i}\right)$, and $v_{i}$ for the same subset of selected observations. Analogously to the previous case, observations with the same value of $I(a)$ are sorted by increasing size of $X_{1 i}$. As before, the highest ranks are almost identical, while the lowest ranks are quite different.

It is also interesting to note that, for the lowest ranks, the difference between the values $u_{i}$ and $v_{i}$ is clearly higher in the left part of Table 5 than in the right part.

Further information on the comparison between the two approaches can be provided by the similarity indices $S_{u}$ and $S_{m}$ introduced in Section 4 and reported in Table 6. By referring to $w_{C Z}$, for the whole population, $S_{u}=0.559$ indicates that only $55.9 \%$ of the units with income below the poverty line are also poor according to the multidimensional approach, while $S_{m}=0.889$ indicates that $88.9 \%$ of the poor units on the basis of $I$ are also poor on the basis of income. Even analyzing particular subsets, a difference between the two methods is confirmed: for the poorest $5 \%$ of the population, $S_{u}=0.775$ implies that over $20 \%$ of the poorest on the basis of income are not poor in the multidimensional approach.

The values of the similarity indices $S_{u}$ and $S_{m}$ obtained by using $w_{e}$ or $w_{U N}$ confirm the previous difference between the indices, with $S_{u}$ steadily lower than $S_{m}$.

Table 6 Similarity indices $S_{u}$ and $S_{m}$ - Italian households 2016 .

\begin{tabular}{lllllll}
\hline \multirow{\alpha}{\alpha}{} & $\begin{array}{l}S_{u} \\
w_{e}\end{array}$ & $S_{m}$ & $\begin{array}{l}S_{u} \\
w_{U N}\end{array}$ & $S_{m}$ & $\begin{array}{l}S_{u} \\
w_{C Z}\end{array}$ & $S_{m}$ \\
\hline 0.05 & 0.841 & 0.978 & 0.771 & 0.948 & 0.775 & 0.975 \\
0.10 & 0.767 & 0.941 & 0.686 & 0.887 & 0.667 & 0.941 \\
0.15 & 0.717 & 0.908 & 0.656 & 0.811 & 0.621 & 0.889 \\
1.00 & 0.671 & 0.867 & 0.616 & 0.754 & 0.559 & 0.889 \\
\hline
\end{tabular}

From Tables 5 and 6 we get a first indication that unidimensional and multidimensional approaches seem to define two different sets of poor households: a more complete analysis, carried out on the basis of rank correlation, is illustrated in the following.

\subsection{Rank correlation analysis}

The highlight of the comparison between the unidimensional and the multidimensional approach is the rank correlation analysis, both overall, and in reference to subsets of the poorest.

The first results refer to the correlation between the multidimensional poverty index $I$ and the equivalent income $X_{1}$. Table 7 shows the indices of Bravais-Pearson $r$, Kendall $\tau$, Spearman $S$ and Gini $G$ Pearson; the first column reports selected values of the cumulative distribution of $I$, where $I$ is in decreasing order, and the 
following columns show the values of the four correlation indices with respect to the three weighting systems used. The last rows of the Table contains the indices for the whole population $(F(I)=100)$, while the first row refers to the poorest $5 \%$ of total population $(F(I)=5)$.

Moving from the bottom to the top of the Table it is clearly observable a strong decrease in rank correlation, thus suggesting that multidimensional and unidimensional approaches define two different subsets of poor units.

Kendall's and Gini's measures give quite similar results and report a lower correlation between $u_{i}$ and $v_{i}$ than Bravais-Pearson and Spearman indices. The United Nations weighting system indicates the lowest values, especially for the poorest subgroups, while $w_{e}$ and $w_{C Z}$ provide substantially similar results. All indices, however, clearly signal a decreasing rank correlation for decreasing values of $F(I)$.

Table 7 Rank correlation between multidimensional poverty index $I$ and equivalent income $X_{1}$ Italian households 2016.

\begin{tabular}{|c|c|c|c|c|c|c|c|c|c|c|c|c|}
\hline \multirow[b]{2}{*}{$F(I)$} & \multicolumn{4}{|c|}{$w_{e}$} & \multicolumn{4}{|c|}{$w_{U N}$} & \multicolumn{4}{|c|}{$w_{C Z}$} \\
\hline & $\mathrm{r}$ & $\tau$ & $S$ & $\mathrm{G}$ & $\mathrm{r}$ & $\tau$ & $\mathrm{S}$ & $\mathrm{G}$ & $\mathrm{r}$ & $\tau$ & $S$ & $\mathrm{G}$ \\
\hline 5 & 0.227 & 0.178 & 0.334 & 0.168 & 0.157 & 0.117 & 0.285 & 0.119 & 0.229 & 0.164 & 0.328 & 0.159 \\
\hline 10 & 0.383 & 0.232 & 0.504 & 0.259 & 0.299 & 0.153 & 0.439 & 0.199 & 0.375 & 0.236 & 0.492 & 0.259 \\
\hline 25 & 0.484 & 0.350 & 0.568 & 0.353 & 0.387 & 0.258 & 0.481 & 0.283 & 0.469 & 0.347 & 0.555 & 0.339 \\
\hline 50 & 0.691 & 0.514 & 0.705 & 0.552 & 0.603 & 0.433 & 0.618 & 0.471 & 0.677 & 0.504 & 0.689 & 0.543 \\
\hline 100 & 0.775 & 0.589 & 0.768 & 0.654 & 0.725 & 0.534 & 0.717 & 0.601 & 0.765 & 0.577 & 0.755 & 0.649 \\
\hline
\end{tabular}

Also in the case of correlation analysis, the robustness of the results is assessed by analyzing the correlation between simulated multidimensional indices $I$ and equivalent income $X_{1}$. Table 8 summarizes the results of 30,000 simulated indices $(10,000$ for each of the 3 weighting systems), showing the average of the correlations obtained.

The resulting picture closely resembles that outlined in Table 7, with the confirmation of all the main results and, above all, of the decreasing correlation for decreasing values of $F(I)$.

Table 8 Rank correlation between simulated multidimensional poverty index and equivalent income $X_{1}$, average of 10000 simulations - Italian households 2016.

\begin{tabular}{|c|c|c|c|c|c|c|c|c|c|c|c|c|}
\hline & & $w_{e}$ & & & & $w_{U N}$ & & & & $w_{C Z}$ & & \\
\hline$F(I)$ & $r$ & $\tau$ & S & G & $r$ & $\tau$ & S & G & $r$ & $\tau$ & $S$ & G \\
\hline 5 & 0.202 & 0.151 & 0.314 & 0.147 & 0.140 & 0.097 & 0.274 & 0.098 & 0.224 & 0.158 & 0.329 & 0.161 \\
\hline 10 & 0.384 & 0.230 & 0.505 & 0.262 & 0.318 & 0.165 & 0.455 & 0.214 & 0.401 & 0.255 & 0.513 & 0.282 \\
\hline 25 & 0.499 & 0.360 & 0.582 & 0.364 & 0.418 & 0.283 & 0.508 & 0.305 & 0.520 & 0.387 & 0.600 & 0.377 \\
\hline 50 & 0.643 & 0.469 & 0.659 & 0.511 & 0.563 & 0.400 & 0.580 & 0.435 & 0.671 & 0.496 & 0.686 & 0.536 \\
\hline 100 & 0.748 & 0.560 & 0.739 & 0.630 & 0.701 & 0.512 & 0.692 & 0.577 & 0.757 & 0.569 & 0.747 & 0.642 \\
\hline
\end{tabular}


In the last step of the rank correlation analysis we compare the rankings derived by the simulated multidimensional indices and by $I$. As before, Table 9 summarizes the results of 30,000 simulated indices (10,000 for each of the 3 weighting systems), showing the average of the correlations obtained. From Table 9 we can observe extremely high values, which suggest that the subsets of poor units identified by the simulated indices are almost coincident, lending further support to the robustness of the multidimensional fuzzy indicator.

Table 9 Rank correlation between simulated multidimensional poverty index and multidimensional poverty index $I$, average of 10000 simulations- Italian households 2016.

\begin{tabular}{|c|c|c|c|c|c|c|c|c|c|c|c|c|}
\hline \multirow[b]{2}{*}{$F(I)$} & \multicolumn{3}{|c|}{$w_{e}$} & \multirow[b]{2}{*}{$\mathrm{G}$} & \multicolumn{4}{|c|}{$w_{U N}$} & \multicolumn{4}{|c|}{$w_{C Z}$} \\
\hline & $r$ & $\tau$ & $S$ & & $\mathrm{r}$ & $\tau$ & $S$ & G & $\mathrm{r}$ & $\tau$ & $\mathrm{S}$ & $\mathrm{G}$ \\
\hline 5 & 0.974 & 0.847 & 0.702 & 0.830 & 0.979 & 0.871 & 0.654 & 0.833 & 0.979 & 0.869 & 0.730 & 0.849 \\
\hline 10 & 0.973 & 0.846 & 0.621 & 0.811 & 0.980 & 0.871 & 0.612 & 0.828 & 0.979 & 0.867 & 0.660 & 0.838 \\
\hline 25 & 0.970 & 0.842 & 0.697 & 0.824 & 0.979 & 0.866 & 0.739 & 0.861 & 0.976 & 0.862 & 0.701 & 0.834 \\
\hline 50 & 0.973 & 0.852 & 0.913 & 0.884 & 0.981 & 0.874 & 0.929 & 0.905 & 0.982 & 0.881 & 0.932 & 0.909 \\
\hline 100 & 0.981 & 0.867 & 0.967 & 0.932 & 0.988 & 0.893 & 0.947 & 0.943 & 0.988 & 0.895 & 0.997 & 0.959 \\
\hline
\end{tabular}

Overall rank correlation analysis results point out to two different subsets of poor units derived from the unidimensional and the multidimensional indicators, suggesting that the two approaches differ not only theoretically, but also as regards data analysis and empirical findings.

\section{Conclusions}

The comparison between unidimensional and multidimensional approach to poverty measurement is carried out by means of a rank correlation analysis, aimed at evaluating the intersection between the set of poor units indicated by the two methods.

The robustness of the comparison is analyzed through a Monte Carlo study, with particular emphasis on the issues related to the choice of $\mu(X)$ and their effects on the set of poor units. The simulated values suggest an extremely satisfactory robustness of the fuzzy poverty indicators, thus overcoming the objection related to the subjectivity of the choice of $\mu(X)$. Furthermore, the simulation study proves to be a particularly powerful tool to investigate further characteristics of the multidimensional fuzzy poverty indicators, such as the weighting system. The main drivers of poverty, identified and ranked on 2016 Italian household data, are educational achievement, dimension of the residence, job status and equivalent income, and they remain remarkably stable over the simulations.

A key point in poverty analyses is not to establish how many are the poor households, but who are they. We contribute to poverty measurement by comparing the 
sets of poor units identified by traditional unidimensional, fuzzy unidimensional and fuzzy multidimensional indicators.

The result of rank correlation analysis allow to demonstrate that the two approaches define two different sets of poor households, and we also show how this difference increases as poorer population subgroups are targeted. Overall our results suggest not only two different theoretical frameworks, but also mismatched empirical findings, with the unidimensional indicator providing only partial information on poverty condition.

Relying on our conclusions, any socio-economic policy to reduce poverty developed on the basis of income information is likely to no achieve its proposed goals, being addressed to socioeconomic units which are, in effect, non-poor. Only in the framework of the multidimensional approach it is possible to correctly individuate the set of the poor and to formulate actions able to reduce poverty.

\section{Appendix}

Table 10 Indicator function $\mu\left(X_{1}\right)$ for the I indicator: Household equivalent disposable income

\begin{tabular}{ll}
\hline & $\mu\left(X_{1}\right)$ \\
\hline$x_{1 i}<0.4 \bar{x}_{1 m e}$ & 1 \\
$0.4 \bar{x}_{1 m e} \leq x_{1 i}<0.6 \bar{x}_{1 m e}$ & 0.9 \\
$0.6 \bar{x}_{1 m e} \leq x_{1 i}<0.8 \bar{x}_{1 m e}$ & 0.5 \\
$0.8 \bar{x}_{1 m e} \leq x_{1 i}$ & 0 \\
\hline
\end{tabular}

\section{References}

1. Aaberge, R., Brandolini, A.: Multidimensional poverty and inequality. Bank of Italy Working Papers, 976, 1-133 (2014)

2. Alkire, S., Santos, M.E.: Measuring acute poverty in the developing world: robustness and scope of the multidimensional poverty index. World Development, 59, 251-274 (2014)

3. Andreoli, F., Zoli, C.: From unidimensional to multidimensional inequality: a review. Metron, 78, 5-42 (2020)

4. Betti, G., Cheli, B., Gambini, R.: A statistical model for the dynamics between two fuzzy states: theory and an application to poverty analysis. Metron, 62, 391-411 (2004)

5. Bossert, W., Chakravarty, S. R., DAmbrosio, C.: Multidimensional poverty and material deprivation. Review of Income and Wealth, 59, 29-43 (2013)

6. Cerioli, A., Zani, S.: A fuzzy approach to the measurement of poverty. In Dagum, C., Xenga, M.: Income and wealth distribution, inequality and poverty. Springer, Berlin, 272-284 (1990)

7. Cheli, B., Lemmi, A.: A totally fuzzy and relative approach to the multidimensional analysis of poverty. Economic Notes, 24, 115-134 (1995) 
Table 11 Indicator function $\mu\left(X_{2}\right)$ for the II indicator: Ratio between the number of the household members perceiving an income and the household size

\begin{tabular}{lll}
\hline Household size & Value of the ratio & $\mu\left(X_{2}\right)$ \\
\hline 1 & 0 & 1 \\
1 & 1 & 0 \\
2 & 0 & 1 \\
2 & $\geq 0.50$ & 0 \\
3 & 0 & 1 \\
3 & $\geq 0.33$ & 0 \\
4 & 0 & 1 \\
4 & 0.25 & 0.4 \\
4 & $\geq 0.50$ & 0 \\
5 & 0 & 1 \\
5 & 0.20 & 0.5 \\
5 & $\geq 0.40$ & 0 \\
6 & 0 & 1 \\
6 & $1 / 6$ & 0.75 \\
6 & $2 / 6$ & 0.25 \\
6 & $\geq 0.50$ & 0 \\
$\geq 7$ & 0 & 1 \\
$\geq 7$ & $0.14-0.29$ & 0.75 \\
$\geq 7$ & $0.30-0.58$ & 0.25 \\
$\geq 7$ & $>0.58$ & 0 \\
\hline
\end{tabular}

Table 12 Indicator function $\mu\left(X_{3}\right)$ for the III indicator: Occupancy title and value of the household residence

\begin{tabular}{llll}
\hline & \multicolumn{3}{l}{ Value } \\
Occupancy title & $>150000$ & $75000-150000$ & $\leq 75000$ \\
\hline Owned & 0 & 0 & 0.5 \\
Rented & 0 & 0.3 & 1 \\
Occupied under redemtion agreement & 0 & 0.2 & 0.5 \\
Occupied in usufruct & 0 & 0.2 & 0.5 \\
Occupied free of charge & 0 & 0.3 & 1 \\
\hline
\end{tabular}

Table 13 Indicator function $\mu\left(X_{4}\right)$ for the IV indicator: Educational achievement of the household head and his/her father

\begin{tabular}{llllll}
\hline \multirow{2}{*}{ Father } & None & $\begin{array}{l}\text { Elementary } \\
\text { school }\end{array}$ & Jead of the household \\
& & 0.6 & 0.2 & 0 & 0 \\
\hline None & 1 & 1 & 0.5 & 0 & 0 \\
Elementary school & 1 & 1 & 1 & 0 & 0 \\
Junior high school & 1 & 1 & 1 & 0.4 & 0 \\
Senior high school & 1 & 1 & 1 & 0.5 & 0 \\
University degree & 1 & 1 & & & \\
\hline
\end{tabular}


Table 14 Indicator function $\mu\left(X_{5}\right)$ for the $\mathrm{V}$ indicator: Educational achievement of the household spouse and her/his father

\begin{tabular}{|c|c|c|c|c|c|}
\hline \multirow[b]{2}{*}{ Father } & \multicolumn{5}{|c|}{ Head of the household } \\
\hline & None & $\begin{array}{l}\text { Elementary } \\
\text { school }\end{array}$ & J. high school & S. high school & University \\
\hline None & 1 & 0.6 & 0.2 & 0 & 0 \\
\hline Elementary school & 1 & 1 & 0.5 & 0 & 0 \\
\hline Junior high school & 1 & 1 & 1 & 0 & 0 \\
\hline Senior high school & 1 & 1 & 1 & 0.4 & 0 \\
\hline University degree & 1 & 1 & 1 & 0.5 & 0 \\
\hline
\end{tabular}

Table 15 Indicator function $\mu\left(X_{6}\right)$ for the VI indicator: Gender, age and occupational status of the household head

\begin{tabular}{llll}
\hline & \multicolumn{3}{l}{ age } \\
& $<25$ & $25-65$ & \multicolumn{2}{l}{65} \\
\hline Male employed head of the household & 0 & 0 & 0 \\
Male unemployed head of the household, employed spouse & 0.4 & 0.2 & 0.3 \\
Male unemployed head of the household, unemployed spouse & 1 & 1 & 1 \\
Male unemployed head of the household, no spouse & 1 & 1 & 1 \\
Female employed head of the household, employed spouse & 0 & 0 & 0 \\
Female employed head of the household, unemployed spouse & 0.2 & 0.5 & 0.2 \\
Female employed head of the household, no spouse & 0 & 0 & 0 \\
Female employed head of the household, no spouse, with children & 0.5 & 0.2 & 0.5 \\
Female unemployed head of the household, employed spouse & 0.5 & 0.3 & 0.4 \\
Female unemployed head of the household, unemployed spouse & 1 & 1 & 1 \\
Female unemployed head of the household, no spouse & 1 & 1 & 1 \\
\hline
\end{tabular}

Table 16 Indicator function $\mu\left(X_{7}\right)$ for the VII indicator: Geographical area of residence

\begin{tabular}{ll}
\hline & $\mu\left(X_{7}\right)$ \\
\hline north & 0 \\
center & 0 \\
south & 0.4 \\
\hline
\end{tabular}

Table 17 Indicator function $\mu\left(X_{8}\right)$ for the VIII indicator: Household size and number of bathrooms of the residence

\begin{tabular}{lll}
\hline Household size & Number of bathrooms & $\mu\left(X_{8}\right)$ \\
\hline whatever & $\geq 2$ & 0 \\
1 & 1 & 0 \\
2 & 1 & 0 \\
3 & 1 & 0.3 \\
4 & 1 & 0.6 \\
$\geq 5$ & 1 & 1 \\
\hline
\end{tabular}


Table 18 Indicator function $\mu\left(X_{9}\right)$ for the IX indicator: Household size and dimension (in square meters) of the residence

\begin{tabular}{lll}
\hline Household size & Square meters & $\mu\left(X_{9}\right)$ \\
\hline 1 & $<50$ & 1 \\
1 & $50-65$ & 0.5 \\
1 & $65-80$ & 0.25 \\
1 & $>80$ & 0 \\
2 & $<60$ & 1 \\
2 & $60-75$ & 0.5 \\
2 & $75-90$ & 0.25 \\
2 & $>90$ & 0 \\
3 & $<70$ & 1 \\
3 & $70-85$ & 0.5 \\
3 & $85-100$ & 0.25 \\
3 & $>100$ & 0 \\
4 & $<80$ & 1 \\
4 & $80-95$ & 0.5 \\
4 & $95-110$ & 0.25 \\
4 & $>110$ & 0 \\
$\geq 5$ & $<100$ & 1 \\
$\geq 5$ & $100-120$ & 0.5 \\
$\geq 5$ & $120-140$ & 0.25 \\
$\geq 5$ & $>140$ & 0 \\
\hline
\end{tabular}

Table 19 Indicator function $\mu\left(X_{1} 0\right)$ for the $\mathrm{X}$ indicator: Professional occupation of the household head

\begin{tabular}{ll}
\hline & $\mu\left(X_{1} 0\right)$ \\
\hline Teacher & 0 \\
Manager & 0 \\
Self employed & 0 \\
Office worker & 0.2 \\
Blue-collar worker & 0.3 \\
Unemployed & 1 \\
Job pensioner & 0.2 \\
Non-job pensioner & 1 \\
\hline
\end{tabular}

8. Dagum, C., Costa, M.: Analysis and measurement of poverty. Univariate and multivariate approaches and their policy implications. In Dagum, C., Ferrari, G.: Household Behaviour, Equivalence Scales, Welfare and Poverty. Springer, Berlin, 221-271 (2004)

9. Deutsch, J., Silber, J.: Measuring multidimensional poverty: an empirical comparison of various approaches. Review of Income and Wealth, 51, 145-174 (2005)

10. Duclos, J.Y., Sahn, D.E., Younger, S.D.: Robust multidimensional poverty comparisons. The Economic Journal, 116, 943-968 (2006)

11. Esposito, L., Chiappero Martinetti, E.: Eliciting, applying and exploring multidimensional welfare weights: evidence from the field. Review of Income and Wealth, 65 (2019)

12. Haagenars, A.J.M.: The perception of poverty. North Holland, Amsterdam (1986)

13. Lemmi, A., Betti, G.: Fuzzy set approach to multidimensional poverty measurement, Springer, Berlin (2006) 
14. Ravallion, M.: On multidimensional indices of poverty. Journal of Economic Inequality, 9, 235-248 (2011)

15. Sen, A.K.: Inequality rexamined. Harvard University Press, Cambridge (MA) (1992)

16. UNPD: Human Development ReportInequality rexamined. Harvard University Press, Cambridge (MA) (1992) 


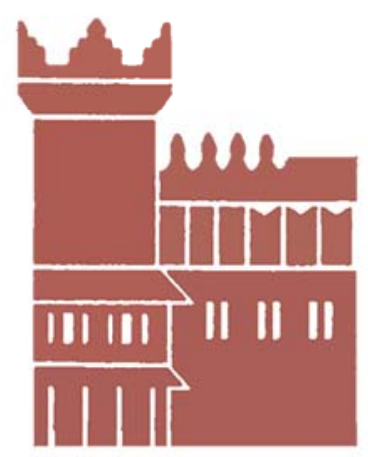

Alma Mater Studiorum - Università di Bologna DEPARTMENT OF ECONOMICS

Strada Maggiore 45

40125 Bologna - Italy

Tel. +39051 2092604

Fax +390512092664

http://www.dse.unibo.it 